\title{
String Theory, the Dark Sector, and the Hierarchy Problem
}

\author{
Per Berglund, ${ }^{1}$ Tristan Hübsch, ${ }^{2}$ and Djordje Minic ${ }^{3}$ \\ ${ }^{1}$ Department of Physics and Astronomy, University of New Hampshire, Durham, NH 03824, USA \\ ${ }^{2}$ Department of Physics and Astronomy, Howard University, Washington, DC 20059, USA \\ ${ }^{3}$ Department of Physics, Virginia Tech, Blacksburg, VA 24061, USA
}

Abstract

We discuss dark energy, dark matter, and the hierarchy problem in the context of a general noncommutative formulation of string theory. In this framework, dark energy is generated by the dynamical geometry of the dual spacetime while dark matter, on the other hand, comes from the degrees of freedom dual to the visible matter. This formulation of string theory is sensitive to both the IR and UV scales, and the Higgs scale is radiatively stable by being a geometric mean of radiatively stable UV and IR scales. We also comment on various phenomenological signatures of this novel approach to dark energy, dark matter, and the hierarchy problem. We find that this new view on the hierarchy problem is realized in a toy model based on a nonholomorphic deformation of the stringy cosmic string. Finally, we discuss a proposal for a new nonperturbative formulation of string theory, which sheds light on M-theory and F-theory, as well as on supersymmetry and holography.

Keywords: string theory, dark energy, dark matter, hierarchy problem

DOI: 10.31526/LHEP.2021.186

\section{INTRODUCTION}

The deep foundations and real world implications of string theory [1] are still shrouded in mystery. In particular, the problem of dark energy has excited a lot of recent discussion within various aspects of string theory $[2,3,4]$. Motivated in part by some prescient work on various aspects of string theory $[5,6,7,8]$, as well as some recent developments in the noncommutative field theory [9], double field theory [10], quantum gravity [11], and quantum foundations [12, 13], a generic, noncommutatively generalized geometric phase-space formulation of string theory [14] has been recently developed. Within this framework, which clarifies many foundational issues found in the classic textbook discussion of string theory [1], it was recently shown that dark energy is naturally induced from the overall curvature of the dual of the observed spacetime [15]. This effect is the leading (zeroth) order term in an expansion of the noncommutative scale, $\lambda$, and is realized in certain stringy-cosmicstring-like toy models that rely only on the ubiquitous axiondilaton ("axilaton") system and gravity [16]. In particular, it was pointed out that the Higgs scale is radiatively stable by being the geometric mean of the radiatively stable UV (Planck) and IR (dark energy) scales, respectively.

In this paper, we consider the inclusion of the dual matter which, to first order in $\lambda$, can be interpreted as the dark matter as well as providing an intrinsic stringy resolution to the hierarchy problem. Furthermore, in this new approach, the known Standard Model fields and their dark matter duals are dynamically correlated, and we comment on various phenomenolog- ical signatures stemming from this correlation. We also comment about other phenomenological signatures of this new approach to dark energy, dark matter, and the hierarchy problem in the context of string theory. In particular, we discuss this new view on the hierarchy problem within a toy model based on a nonholomorphic deformation of the stringy cosmic string. Finally, we present a proposal for a new nonperturbative formulation of string theory, which sheds light on M-theory as well as F-theory and illuminates the emergence of supersymmetry and holography.

\section{GENERAL STRING THEORY AND DARK ENERGY}

We begin our discussion with a review of the generic noncommutative and doubled formulation of string theory and its relation to dark energy. Following the recent discussion in [14], the starting point is the chiral string worldsheet description

$$
S_{\mathrm{str}}^{\mathrm{ch}}=\frac{1}{4 \pi} \int_{\tau, \sigma}\left[\partial_{\tau} \mathbb{X}^{A}\left(\eta_{A B}+\omega_{A B}\right)-\partial_{\sigma} \mathbb{X}^{A} H_{A B}\right] \partial_{\sigma} \mathbb{X}^{B},
$$

with $\tau, \sigma$ worldsheet coordinates. Furthermore, $\mathbb{X}^{A}(\tau, \sigma),(A=$ $1, \ldots, 26$, for the critical bosonic string) combine the sum $\left(x^{a}\right)$ and the difference $\left(\tilde{x}_{a}\right)$ of the left- and right-moving chiral bosons on the string. ${ }^{1}$ The mutually compatible dynamical fields are the antisymmetric symplectic structure $\omega_{A B}$, the symmetric polarization metric $\eta_{A B}$, and the doubled symmetric metric $H_{A B}$, respectively, defining the so-called Born geometry [14]. Quantization renders the doubled "phase-space" operators $\hat{\mathbb{X}}^{A}=$

\footnotetext{
${ }^{1}$ This formulation also leads to a natural proposal for a nonperturbative string theory which will be discussed in Section 7 of this paper.
} 
$\left(\hat{x}^{a} / \lambda, \hat{\tilde{x}}_{a} / \lambda\right)$ inherently noncommutative, inducing [14]

$$
\left[\hat{\mathbb{X}}^{A}, \hat{\mathbb{X}}^{B}\right]=i \omega^{A B}
$$

or, in components, for constant nonzero $\omega^{A B}$,

$$
\left[\hat{x}^{a}, \hat{\tilde{x}}_{b}\right]=2 \pi i \lambda^{2} \delta_{b}^{a}, \quad\left[\hat{x}^{a}, \hat{x}^{b}\right]=0=\left[\hat{\tilde{x}}_{a}, \hat{\tilde{x}}_{b}\right]
$$

where $\lambda$ denotes the fundamental length scale, such as the fundamental Planck scale, $\epsilon=1 / \lambda$ is the corresponding fundamental energy scale, and the string tension is $\alpha^{\prime}=\lambda / \epsilon=\lambda^{2}$; see also Section 6. Note that the Hamiltonian and diffeomorphism constraints, $\partial_{\sigma} \mathbb{X}^{A} H_{A B} \partial_{\sigma} \mathbb{X}^{B}=0$ and $\partial_{\sigma} \mathbb{X}^{A} \eta_{A B} \partial_{\sigma} \mathbb{X}^{B}=$ 0 , respectively, are treated on the equal footing. In particular, the usual spacetime interpretation of the zero-mode sector of string theory [1] is tied to the solution of the diffeomorphism constraint by level matching. In this more general and generically noncommutative formulation, the spacetime interpretation is replaced by a modular spacetime (or quantum spacetime) realization (also found in the context of quantum foundations) [14]. Thus, all effective fields must be regarded as bilocal $\phi(x, \tilde{x})[14]$, subject to (3), and therefore inherently non-local in the conventional $x^{a}$-spacetime. Such noncommutative field theories [9] generically display a mixing between the ultraviolet (UV) and infrared (IR) physics with continuum limits defined via a double-scale renormalization group (RG) and the self-dual fixed points [9], [14]. This has profound implications for the generic physics of string theory and, in particular, the problems of dark energy, dark matter, and the separation of scales that goes beyond the realm of effective field theory.

In [15], we have argued that the generalized geometric formulation of string theory discussed above provides for an effective description of dark energy that is consistent with a de Sitter spacetime. This is due to the theory's chirally and noncommutatively (3) doubled realization of the target space and the stringy effective action on the doubled noncommutative (3) spacetime $\left(x^{a}, \tilde{x}_{a}\right)$

$$
S_{\mathrm{eff}}^{n c}=\iint \operatorname{Tr} \sqrt{g(x, \tilde{x})}\left[R(x, \tilde{x})+L_{m}(x, \tilde{x})+\ldots\right],
$$

including the matter Lagrangian $L_{m}$ and with the corresponding Planck lengths set to one. (The ellipses denote higher-order curvature terms induced by string theory.) This result can be understood as a generalization of the famous calculation by Friedan [17]. Using (3), $S_{\text {eff }}^{n c}$ clearly expands into numerous terms with different powers of $\lambda$, which upon $\tilde{x}$-integration and from the $x$-space vantage point produce various effective terms. Dropping $L_{m}$, for now, to the lowest (zeroth) order of the expansion in the noncommutative parameter $\lambda$ of $S_{\text {eff }}^{n c}$ takes the form

$$
S=-\iint \sqrt{-g(x)} \sqrt{-\tilde{g}(\tilde{x})}[R(x)+\tilde{R}(\tilde{x})],
$$

a remarkable result which was first obtained almost three decades ago by Tseytlin [7], effectively neglecting $\omega_{A B}$ in (2) by assuming that $[\hat{x}, \hat{\tilde{x}}]=0[7]$.

In this leading limit, the $\tilde{x}$-integration in the first term of (5) defines the gravitational constant $G_{N}$ and in the second term produces a positive cosmological constant $\Lambda>0$. Hence, the weakness of gravity is determined by the size of the canonically conjugate dual space, while the smallness of the cosmological constant is given by its curvature. In particular, we have

$$
\bar{S}=\frac{\int_{X} \sqrt{-g(x)}[R(x)+\ldots]}{\int_{X} \sqrt{-g(x)}}+\ldots
$$

which leads to a seesaw-like formula for the cosmological constant, discussed below.

So far, we have omitted the matter sector explicitly. In what follows, we argue that the dual part of the matter sector appears as dark matter, which is, in turn, both sensitive to dark energy [18] and also dynamically correlated with the visible matter. We next focus on this dark matter and emphasize the unity of the description of the entire dark energy and matter sector, induced and determined by the properties of the dual spacetime, as predicted by this general, noncommutatively phase-space doubled formulation of string theory.

\section{DARK SECTOR AND THE HIERARCHY PROBLEM}

We have already emphasized that in this generic noncommutative formulation of string theory, all effective fields must be regarded a priori as bilocal $\phi(x, \tilde{x})[14]$, subject to (3). Moreover, the fields are doubled as well, and thus for every $\phi(x, \tilde{x})$, there exists a dual $\tilde{\phi}(x, \tilde{x})$. This can be easily seen in the background field approach, which we will consider in the next section. Therefore, in general, to the lowest (zeroth) order of the expansion in the noncommutative parameter, $\lambda S_{\text {eff }}^{n c}$ takes the following form (that also includes the matter sector and its dual), which generalizes equation (5) (and where, once again, the corresponding Planck lengths are set to one):

$$
\iint \sqrt{g(x) \tilde{g}(\tilde{x})}\left[R(x)+\tilde{R}(\tilde{x})+L_{m}(A(x, \tilde{x}))+\tilde{L}_{d m}(\tilde{A}(x, \tilde{x}))\right] .
$$

Here, the $A$ fields denote the usual Standard Model fields, and the $\tilde{A}$ fields are their duals, as predicted by the general formulation of quantum theory that is sensitive to the minimal length (the noncommutative parameter $\lambda$ [14]). In the following section, we will elaborate more explicitly on the dual matter degrees of freedom. Right now, we are concerned with the generalization of the discussion summarized in the previous section.

After integrating over the dual spacetime, and after taking into account T-duality, equation (6) now reads: ${ }^{2}$

$$
\bar{S}^{\prime}=\frac{\int_{X} \sqrt{-g(x)}\left[R(x)+L_{m}(x)+\tilde{L}_{d m}(x)\right]}{\int_{X} \sqrt{-g(x)}}+\ldots
$$

The proposal here is that the dual sector (as already indicated in the previous section) should be interpreted as the dark matter

\footnotetext{
${ }^{2}$ Tseytlin proposal has been further explored by Davidson and Rubin who in particular showed that the cosmological constant is necessarily nonnegative definite [19].
} 
sector, which is correlated with the visible sector via the dark energy sector, as discussed in [18]. We emphasize the unity of the description of the entire dark sector based on the properties of the dual spacetime, as predicted by the generic formulation of string theory (as a quantum theory with a dynamical Born geometry) [14].

Let us turn off the dynamical part of gravity and consider the hierarchy problem. First, we have

$$
S_{0}=-\iint \sqrt{g(x) \tilde{g}(\tilde{x})}\left[L_{m}(A(x, \tilde{x}))+\tilde{L}_{d m}(\tilde{A}(x, \tilde{x}))\right],
$$

which leads to the following nonextensive action to lowest order in $\Lambda$

$$
\bar{S}_{0}=\frac{\int_{X} \sqrt{-g(x)}\left[L_{m}(x)+\tilde{L}_{d m}(x)\right]}{\int_{X} \sqrt{-g(x)}}+\ldots
$$

after integrating over the dual spacetime.

This implies a seesaw formula which involves the matter scale from the matter (and dark matter) part of the action and the scales related to the UV (Planck scale, $M_{P}$ ) and the IR (dark energy scale, $M_{\Lambda}$ ):

$$
M_{\Lambda} M_{P} \sim M_{H}^{2},
$$

where $M_{H}$ denotes the characteristic matter scale. Completely generally, this relation follows from the diffeomorphism constraint of the chiral string worldsheet, which is controlled by the $O(d, d)$ biorthogonal metric $\eta$ and which implies, in the limit of zero modes (and zero momenta), that $E \tilde{E}=M^{2}$. Here, $E$ and $\tilde{E}$ are the energy scales in the observed and dual spacetime, respectively, and $M$ is a new (mass) parameter. Since the IR (dark energy) scale can be interpreted as the vacuum energy, which in the matter sector is controlled by the Higgs potential, $M$ naturally sets the Higgs scale, $M_{H}$, which is indeed the case numerically. If we remember that the geometry of the dual spacetime is responsible for the origin of dark energy, then the dual energy $\tilde{E}$ can be set to the dark energy scale. Then, the fundamental energy scale $E$ in the observed spacetime is the Planck scale. This mixing of the UV and IR scales in a fully covariant formulation is a unique feature of the chiral string worldsheet theory. (For other approaches to the hierarchy problem which mix the UV and IR scales but which violate covariance, consult [20].)

Note that both the UV and IR scales are radiatively stable. First, we note that the $M_{P}$ is the UV scale and the issue of radiative stability does not apply to it. Second, let us recall the radiative stability of the dark energy scale $M_{\Lambda}$, the IR scale: in particular, as we have noticed, in our previous work, the effective action of the sequester type [21] (see also [22])

$$
\int_{x} \sqrt{-g}\left[\frac{R}{2 G}+s^{4} L\left(s^{-2} g^{a b}\right)+\frac{\Lambda}{G}\right]+\sigma\left(\frac{\Lambda}{s^{4} \mu^{4}}\right),
$$

where $L$ denotes the combined Lagrangians for the matter and dark matter sectors, $\mu$ is a mass scale, and $\sigma\left(\frac{\Lambda}{s^{4} \mu^{4}}\right)$ is a global interaction that is not integrated over [21]. This can be provided by our set-up: start with bilocal fields $\phi(x, \tilde{x})[14]$, and replace the dual labels $\tilde{x}$ and also $\lambda$ (in a coarsest approximation) by the global dynamical scale $s \sim \Delta \tilde{x} \sim \lambda^{2} \Delta x^{-1}$. Also, normal ordering produces $\sigma$. This is an effective realization of the sequester mechanism in a noncommutative phase of string theory. One important lesson here is that the low-energy effective description of the generic string theory is, to the lowest order, a sequestered effective field theory and, more generally, a noncommutative effective field theory [9] of a new kind, which is defined within a doubled RG, which is covariant with respect to the UV and IR cutoffs and which is endowed with a self-dual fixed point [14].

\section{DARK MATTER AND STRING THEORY}

In this section, we elaborate on the dual matter degrees of freedom and the explicit appearance of dark matter in the general formulation of string theory [14]. The previous discussion looked at the stringy effective action to the lowest order in $\Lambda$, neglecting the noncommutative aspect of the generalized geometric description of the string worldsheet (1). In order to see the effect of the leading order correction in $\Lambda$ of (7), we consider the zero modes of $S_{\mathrm{str}}^{\mathrm{ch}}$. The associated particle action is fixed by the symmetries of the chiral worldsheet in terms of the symplectic form $\omega$, the $O(D, D)$ metric $\eta$, and the double metric $H$-the so-called Born geometry-and takes the following form (following the general results for the chiral string worldsheet description [14]):

$$
S_{M P}=\int_{\tau}\left(p \dot{x}+\tilde{p} \dot{\tilde{x}}-\lambda^{2} p \dot{\tilde{p}}-N h-\tilde{N} d\right) .
$$

Here, the Hamiltonian constraint, fixed by the double metric, is given by $h=p^{2}+\tilde{p}^{2}+m^{2}$, and the diffeomorphism constraint, fixed by the $O(D, D)$ metric, is $d=p \tilde{p}-M^{2}$. These constraints are inherited from the quantization of chiral worldsheet theory. Finally, the symplectic structure fixes the $\lambda^{2} p \dot{\tilde{p}}$ term. Note that $m^{2}$ should not be confused with the (mass) ${ }^{2}$ of a particle excitation. In parallel with the usual discussion found in introductory chapters of textbooks on quantum field theory, one has to understand the representation theory associated with the symmetries of the underlying Born geometry and interpret $m^{2}$ and $M^{2}$ in terms of the relevant Casimirs in the full representation theory of this description [23]. We are not going to pursue this question in what follows, but we alert the reader that $M$ is a new parameter not found in the context of the effective field theory description, while $m^{2}$ can be interpreted as a particle mass only in a very degenerate limit in which $\tilde{p}=0$ and $M=0$.

For concreteness, let us start with a vector background, by shifting the momenta and the dual momenta by the standard, minimally coupled gauge field and its dual [14]:

$$
p_{a} \rightarrow p_{a}+A_{a}(x, \tilde{x}), \quad \tilde{p}_{a} \rightarrow \tilde{p}_{a}+\tilde{A}_{a}(x, \tilde{x}) .
$$

These gauge fields have the usual Abelian gauge symmetries. Thus, in the target space action for the gauge fields, we end up with canonical Maxwellian terms (with the obvious index structure) plus a characteristic coupling inherited from the 
symplectic structure

$$
\int_{x, \tilde{x}}\left[F^{2}-a \lambda^{2} \llbracket A, \tilde{A} \rrbracket+\tilde{F}^{2}+F \tilde{F}+\ldots\right],
$$

where the $\lambda^{2} \llbracket A, \tilde{A} \rrbracket$ term stems directly from the $\lambda^{2} p \dot{\tilde{p}}$-term in (13) by the "minimal coupling" shift (14), its dimensionful coefficient $a$ to be determined below. This "mixing" term may be expressed as

$$
\begin{aligned}
& \llbracket A, \tilde{A} \rrbracket \stackrel{\text { def }}{=} \int_{\tau_{*}}^{\tau} \mathrm{d} \tau^{\prime} A\left(x\left(\tau^{\prime}\right)\right) \frac{\mathrm{d} \tilde{A}\left(\tilde{x}\left(\tau^{\prime}\right)\right)}{\mathrm{d} \tau^{\prime}}, \\
& =\frac{1}{2}[A(x) \tilde{A}(\tilde{x})]_{\tau_{*}}^{\tau}+\frac{1}{2} \int_{\tau_{*}}^{\tau} \mathrm{d} \tau^{\prime}[A(x) \dot{\tilde{A}}-\dot{A}(x) \tilde{A}(\tilde{x})] .
\end{aligned}
$$

The first of these includes a (worldline-local) mixing term, together with its value at some "reference" proper time, $\tau_{*}$, the integral of which (15) evaluates to an irrelevant additive constant. The second part, $[A \dot{\tilde{A}}-\dot{A} \tilde{A}]$, is far more interesting, as it provides a telltale "Zeemann"-like coupling of $(A, \tilde{A})$-pairs to corresponding "external/background" fluxes, scaled by the coefficient " $a \lambda^{2}$." This relies on identifying $A, \tilde{A}$ as canonical coordinates in the target-spacetime (classical) field theory. Alternatively, in the underlying worldsheet quantum field theory, the $A, \tilde{A}$ are coefficients of certain quantum states, for which the $\llbracket A, \tilde{A} \rrbracket$-term likewise accompanies a Berry's phase-like quantity.

The second quantization of the action (15) in the Coulomb gauge (and its dual) produces the following structure fixed by the Born geometry:

$$
\int_{x, \tilde{x}}\left[(\partial A)^{2}+(\tilde{\partial} A)^{2}-a \lambda^{2} \llbracket A, \tilde{A} \rrbracket+(\tilde{\partial} \tilde{A})^{2}+(\partial \tilde{A})^{2}+\partial A \tilde{\partial} \tilde{A}+\ldots\right] .
$$

Integrating over the dual spacetime $(\tilde{x})$, and setting $\tilde{p} \rightarrow 0$ in the observable spacetime for consistency, poduces (with indices on the respective gauge fields fully restored)

$$
\int_{x}\left[\left(\partial_{[a} A_{b]}\right)^{2}-\frac{\lambda^{8}}{L^{10}} f_{b}^{a} \llbracket A_{a}, \tilde{A}^{b} \rrbracket+\left(\partial_{[a} \tilde{A}_{b]}\right)^{2}+\ldots\right],
$$

where $\tilde{A}_{a} \stackrel{\text { def }}{=} \eta_{a b} \tilde{A}^{b}$ and $f_{b}^{a}$ encode "background fluxes," naturally at the fundamental scale $\lambda$ from (3). Properly normalized on the worldline (17), the "mixing" term $\llbracket A_{a}, \tilde{A}^{a} \rrbracket$ in spacetime must be renormalized by the volume of the primordial observable spacetime, $L^{10}$, prior to any compactification and inflation. This term is thereby sensitive to both the fundamental UV cutoff $\lambda$ and the primordial IR cutoff $L$.

In other words, in the observable spacetime, the visible sector and its dual/dark counterpart are "mixed/correlated" (19), in a way that is sensitive to both the UV and IR cutoffs. This correlation becomes invisible in effective field theory, and it vanishes as either $\lambda \rightarrow 0$ or $L \rightarrow \infty$. Note that with a particular double scaling, this term can be finite! This is consistent with the general set-up of the chiral string worldsheet theory which has two cutoffs (UV and IR) and which generically should be defined with self-dual RG fixed points. Such a correlation between visible and dark matter involving an IR scale (in this case, the Hubble scale) has been observed in astronomical data and has been studied in the context of modified dark matter in [18]. However, the ratio $\frac{\lambda^{8}}{L^{10}}$ should not be naively considered to be trans-Planckian, because that would require that $\lambda$ is the Planck length, and $L$ is the Hubble length. Instead, the two scales should be considered as effective UV and IR scales, the joint appearance of which is a direct evidence of a departure from effective field theory, as further discussed in Section 6.

Having illustrated the general idea with the vector fields $A_{a}$ and $\tilde{A}^{a}$, the form of the corresponding action for a scalar $\phi$ and its dual $\tilde{\phi}$ is immediate (and similarly for pseudo-scalars):

$$
\int_{x}\left[(\partial \phi)^{2}-\frac{\lambda^{8}}{L^{10}} \llbracket \phi, \tilde{\phi} \rrbracket+(\partial \tilde{\phi})^{2}+\ldots\right],
$$

as this would be forced in every dimensional reduction framework. Similarly, the corresponding action for the fermions (by taking the "square root" of the propagating part of the scalar action to accommodate the spin-statistics theorem) is

$$
\int_{x}\left[(\bar{\psi} \partial \psi)-\frac{\lambda^{9}}{L^{10}} \llbracket \bar{\psi}, \tilde{\psi} \rrbracket+(\overline{\tilde{\psi}} \partial \tilde{\psi})+\ldots\right],
$$

where $\llbracket \bar{\psi}, \tilde{\psi} \rrbracket=\bar{\psi} \tilde{\psi}$ are nonderivative bilinear terms, accompanied by "external" fluxes as in (19). This result may be justified by target-spacetime supersymmetry, even if supersymmetry is ultimately broken: the indicated terms are restricted to free fields in flat spacetime. In particular, the omitted interaction terms here also include metric and curvature deviations from flat spacetime. From the underlying worldline, or even worldsheet [14] point of view, such terms are induced from generalizing (13) along the standard construction of GLSMs [24]; the "free-field-limit" terms shown herein however remain unchanged. The same prefactor is also implied from the worldline point of view: the superpartner of each $p \dot{p}$-term in the Lagrangian (13) is a fermionic bilinear, $\bar{\chi} \widetilde{\chi}$, which couples to the same "external/background" flux (20), giving rise to a worldline super-Zeemann effect [25].

The $\frac{\lambda^{9}}{L^{10}}$ scaling coefficient in (21), forced on dimensional grounds in this mass-mixing term, sets the scale in this novel "seesaw mechanism," in principle tunable to induce naturally small neutrino masses. In fact, the inclusion of several mass scales enables concrete models to incorporate an entire hierarchy of seesaw mechanisms, with a more reasonable chance to approach the intricacies of a realistic mass spectrum.

To summarize, the leading "kinetic" parts in the actions (20) and (21), together with the "mixing terms," $\llbracket \phi, \tilde{\phi} \rrbracket$ and $\llbracket \bar{\psi}, \tilde{\psi} \rrbracket$, are seen to be natural: (a) by dimensional reduction from (19) to (20) and (b) by supersymmetry from (20) to (21). Spacetime supersymmetry is broken by interaction terms, explicitly omitted from (19), (20), and (21), such as in the "axilaton" system [16], and not at all unlike the Polonyi mechanism [26]; for a recent discussion, see also [27].

In addition, pseudo-scalars such as axions can be viewed as boost generators (at least for constant profiles) between the observed and dual spacetimes [14]. First, note that the constant 
Kalb-Ramond field can be absorbed into a nontrivial symplectic form (on its diagonal) after an $O(d, d)$ rotation [14]. Thus, the Kalb-Ramond two-form enters into an explicit noncommutativity of the modular spacetime, and it can be used to rotate between observed and dual spacetime coordinates (as an explicit illustration of relative, or observer-dependent, locality) [14]. Since the Kalb-Ramond two-form field dualizes into a pseudo-scalar in $4 \mathrm{~d}$, the $4 \mathrm{~d}$ axion has the same features. More generally, nonconstant Kalb-Ramond profiles imply nonassociative structure [14]. Thus, axions are indicators of noncommutative (when constant) and nonassociative (when propagating) structures in modular spacetime, respectively.

Finally, we note that the peculiar correlation between the visible and dark sectors, discussed for scalar, pseudo-scalar, fermionic, and vector degrees of freedom, can be also found in the gravitational and dual gravitational sectors. Thus, the observed gravity and dark energy are correlated via the scale of noncommutativity. This might have interesting observable effects for the so-called $H_{0}$ tension [28]; see [29].

\section{COMMENTS ON PHENOMENOLOGI- CAL IMPLICATIONS}

The most important general predictions of the chiral string worldsheet theory [14] are as follows: (1) the geometry of the dual spacetime determines the dark energy sector [15], and (2) the dual matter degrees of freedom naturally appear as dark matter candidates, as discussed in the preceding section. We note that, quite explicitly, the dark matter sector provides "sources" for the visible matter sector. This follows from the coupling $\frac{\lambda^{8}}{L^{10}} \llbracket \phi, \tilde{\phi} \rrbracket$, as predicted by the dou$\mathrm{bled} /$ noncommutative set-up, and provides an explicit correlation between the dark matter sector and a visible sector. Given the seesaw formula for the dark energy which relates the dark energy scale to the fundamental length, which could be taken to be the Planck energy scale, then the dark matter is also sensitive to the dark energy. So, the visible matter, dark matter, and dark energy are all related. This is consistent with the observational evidence presented in [18], as we have already alluded to in this article.

We emphasize that in our discussion of the hierarchy problem, the UV and IR scales are radiatively stable, and so is their product, the Higgs scale. This new view on the hierarchy problem goes beyond the usual tools of effective field theory due to explicit presence of the widely separated UV and IR scales. The usual suggested approaches to the hierarchy problem: technicolor, SUSY, and extra dimensions are all within the canonical effective field theory. In the context of string theory, effective field theory (and the approach to the hierarchy problem via a SUSY effective field theory) can be naturally found via string compactifications, but in that case, one is faced with the issue of supersymmetry breaking (and the fundamental question of "measures" on the string landscape/swampland $[2,3,4])$. We claim that these issues are transcended in the general, doubled, and noncommutative formulation of string theory with a fundamentally bosonic and noncommutative formulation, wherein spacetime and matter (and supersymmetry at the Planck scale) can be viewed as emergent phenomena.

Next, we comment on the seesaw formula, which mixes UV and IR scales, and the neutrino sector. Such a seesaw would involve the neutrino and its dual partner. Quite generically, the dual sector acts as a source for the visible sector, and the overall effect is to make the visible sector essentially massive. This immediately provides a curious-and ubiquitousmixing "mass" effect, where the length scale ratio $\lambda^{9} / L^{10}$ may well be phenomenologically relevant; see Section 6 . For every fermion-dual fermion pair, there is a mass matrix of the general form $\left[\begin{array}{cc}m & \lambda^{9} / L^{10} \\ \lambda^{9} / L^{10} & \widetilde{m}\end{array}\right]$, which induces well-known seesaw relations. This includes all Dirac mass terms, as well as a mix of Dirac and Majorana terms, and may well provide a new way of generating neutrino masses.

Next, we comment on other phenomenological issues associated with the dual Standard Model. In the dual QED sector, we should find a dual photon that is correlated with the visible photon and that is distinct from the dark photon of effective field theory. The correlation is proportional to the fundamental length and is finite even in the limit of zero momenta (deep infrared). Also, the usual visible photon/dark photon coupling is subdominant to this term that is inherent in our story. Similarly, in the dual of the weak sector of the Standard Model, we have a dual of the visible $Z$. This dual of $Z$ should be distinguished from the usual $Z^{\prime}$ by its sensitivity to the fundamental length and by its correlation to the visible $Z$. These types of correlations might be found in correlated events in the accelerators, which are not products of any standard particle decays.

Finally, in the dual QCD, we should find interesting phenomenology in the deep infrared, even though that is a very difficult region to study in QCD. In particular, given the new view of the axion field in the above discussion, we have a possible new viewpoint of the strong CP problem in QCD. The first observation here is that, according to [14], the constant KalbRamond field mixes $x$ and $\tilde{x}$ spacetimes (it acts as boost that linearly combines the spacetime and its dual in the context of a larger doubled and noncommutative quantum spacetime). Also, the commutator of dual spacetime coordinates is given by the constant $B$ Kalb-Ramond 2-form field. So, for $B=0$, we get just the observed (4d) spacetime. Also, its $H=\mathrm{d} B$ field strength is trivially zero. But $H$ is dual (in $4 \mathrm{~d}$ ) to the axion (a), which is also constant. But $B$ is zero (there is no preferred background direction), and so, this constant axion may be interpreted as a uniform distribution for the axion (whose constant values can be positive and negative). Now, focus on the QCD axion, relevant to the strong $\mathrm{CP}$ problem, and appearing in the $\mathrm{CP}$ violating term $a F \wedge F$. Averaging this term, linear in the axion, over a uniform distribution for this axion produces zero: $\left(\int_{-k}^{k} \mathrm{~d} a a=0\right.$, with $\left.k \rightarrow \infty\right)$. For a complete argument, we would have to study small fluctuations of the axion field in order to understand the robustness of this new viewpoint on the strong CP problem. 


\section{DARK ENERGY SEESAW AND THE HI- ERARCHY PROBLEM}

The preceding discussion about dark energy, dark matter, and the hierarchy problem is based on the generic noncommutative formulation of string theory. We now present a more conventional realization of the above analysis within a class of a specific discretuum of toy models [30,31, 32, 33, 16] that aim to realize de Sitter space in string theory. In particular, several features of the above noncommutatively generalized phase-space reformulation of string theory are naturally captured due to the essential stringy nature of the models.

This family of models is constructed by starting with an F-theoretic [34] type-IIB string theory spacetime, $W^{3,1} \times Y^{4} \times$ $Y_{\perp}^{2}\left(\times T^{2}\right)$, where the complex structure of the zero-size "hidden" $T^{2}$ fiber of F-theory is identified with the axion-dilaton $\tau \stackrel{\text { def }}{=} \alpha+i e^{-\Phi}$ modulus. Specifically, we compactify on $Y^{4}=\mathrm{K} 3$ or $T^{4}$ and let the observable spacetime $W^{3,1}$ (via warped metric) vary over $Y_{\perp}^{2}$, and $Y_{\perp}^{2} \rightarrow S^{1} \times Z$, with the polar parametrization $\ell e^{z+i \theta}=r e^{i \theta}$, where $z \stackrel{\text { def }}{=} \log (r / \ell) \in Z$, while $Y^{4}$ preserves supersymmetry. Finally, we deform $\tau$ to vary nonholomorphically, only over $S^{1} \subset Y^{2}$. By cross-patching two distinct solutions and by deforming the apparently singular metric into de Sitter space, we get the final nonsupersymmetric solution. The codimension-2 solution $W^{3,1} \rtimes\left(S^{1} \times Z\right)$ has a positive cosmological constant, $\Lambda$, along $W^{3,1}$, and the warped metric is [35]

$$
\mathrm{d} s^{2}=A^{2}(z) \bar{g}_{a b} \mathrm{~d} x^{a} \mathrm{~d} x^{b}-\ell^{2} B^{2}(z)\left(\mathrm{d} z^{2}+\mathrm{d} \theta^{2}\right),
$$

where $\bar{g}_{a b} \mathrm{~d} x^{a} \mathrm{~d} x^{b}=\mathrm{d} x_{0}^{2}-e^{2 \sqrt{\Lambda} x_{0}}\left(\mathrm{~d} x_{1}^{2}+\mathrm{d} x_{2}^{2}+\mathrm{d} x_{3}^{2}\right)$ is the metric on $W^{3,1}$. The two explicit solutions for $\tau$ are [30]

$$
\begin{aligned}
& \tau_{I}(\theta)=b_{0}+i g_{s}^{-1} e^{\omega\left(\theta-\theta_{0}\right)}, \\
& \tau_{I I}(\theta)=\left(b_{0} \pm g_{s}^{-1} \tanh \left[\omega\left(\theta-\theta_{0}\right)\right]\right) \pm i \frac{g_{s}^{-1}}{\cosh \left[\omega\left(\theta-\theta_{0}\right)\right]} .
\end{aligned}
$$

Given the stringy $\mathrm{SL}(2 ; \mathbb{Z})$ monodromy of the axion-dilaton system over a transversal 2-plane $Y_{\perp}^{2}$ in the spacetime, these toy models exhibit S-duality. In generalizations where various moduli fields replace the axion-dilaton system, this directly implies T-duality, which is covariantly realized in the generic phase-space approach (1)-(3).

We emphasize that these models are a deformation of the stringy cosmic string (D7-brane in IIB string theory) [36] and as such represent effective stringy solutions (in the sense of [37]) and not just IIB supergravity solutions. That is, our solutions are indeed found as deformations of certain classic F-theory backgrounds, but as codimension-2 solutions, they can be viewed as effective stringy solutions with an effective "worldsheet" description that is, to lowest order, doubled and generically noncommutative (as described by equation (1)). Thus our deformed stringy cosmic string solutions are naturally equipped with a generalized geometric (and noncommutatively doubled) spacetime structure, which to the lowest order of the doubled target space description directly connects to [7]. Therefore, certain generic features of this doubled description, such as the intensive effective action, directly translate into certain geometric features of our models, discussed below.

In these string models, the cosmological constant within the codimension-2 brane world is determined by the anisotropy $\Delta \omega$ of the axion-dilaton system whose effective energy momentum tensor is given via

$T_{\mu \nu}-\frac{1}{2} g_{\mu \nu} g^{\rho \sigma} T_{\rho \sigma}=\mathcal{G}_{\tau \bar{\tau}} \partial_{\mu} \tau \partial_{\nu} \bar{\tau}=\operatorname{diag}\left[0, \cdots, 0, \frac{1}{4} \omega^{2} \ell^{-2}\right]$,

with $\mathcal{G}_{\tau \bar{\tau}}=-1 /(\tau-\bar{\tau})^{2}$, and where $\ell$ is the characteristic length scale in the transversal 2-plane $Y_{\perp}^{2}[32,33,16]$ :

$$
\Lambda \sim \frac{\Delta \omega^{2}}{\ell^{2}} \text { implies } M_{\Lambda} \sim M^{2} / M_{P}
$$

relating the mass scales of the vacuum energy/cosmological constant $\left(M_{\Lambda}\right)$, particle physics, i.e., Standard Model $(M)$, and the Planck scale $\left(M_{P}\right)$. This seesaw formula can be seen to arise in two ways: first, the formula (29) may be understood as a consequence of dimensional transmutation, whereby the (modified) logarithmic nature of the transversal Green's function [30] (characteristic only of codimension-2 solutions) relates the length scales $\ell$ and $\sqrt{\Lambda}$ [32]. Alternatively, the seesaw formula (29) follows from adapting Tseytlin's result for $\bar{S}$ to the models of $[32,33,16]$ : in the denominator of the above formula, the volume of the transversal 2-plane produces the length scale $\int_{Y_{\perp}^{2}} \sqrt{-g(x)} \propto \ell^{2}$; the numerator (with $\Delta \omega^{2} \stackrel{\text { def }}{=}$ $\left.\left(\omega^{2}-\omega_{c}^{2} A^{2}(z=0)\right)\right)$

$$
\int_{Y_{\perp}^{2}} \sqrt{-g(x)}\left(R(x)+L_{m}\right) \propto \Delta \omega^{2}
$$

reproduces the anisotropy variance of these axion-dilaton profiles, whereas the remaining volume-integration renormalizes the Newton constant as required in [30, 32]. The anisotropy $\omega$ determines the above axion-dilaton stress tensor for the de Sitter solution and asymptotes to the Minkowski cosmic brane limit $\omega_{c}$ at $z \rightarrow 0$. Note that in the F-theory limit, $\omega \rightarrow 0$ and $\omega_{c} \rightarrow 0$. This singular supersymmetric configuration is deformed into a de Sitter background by turning on an anisotropic axion-dilaton profile (23)-(24). Thus, $\Lambda$ that figures in the seesaw formula can be understood as being related to the cosmological breaking of supersymmetry. We stress that our discussion gives an argument for the existence of de Sitter background in string theory, albeit in its generic generalized geometric and intrinsically noncommutative formulation, which from the effective spacetime description is described by our stringy models. One of the features of this doubled and generalized geometric description is that the effective spacetime action is intensive (as opposed to extensive), which directly translates into the seesaw formula for the cosmological constant (29).

Note that more explicitly

$$
\Lambda_{D-2} \sim \frac{\Delta \omega^{2}}{\ell^{2}}=\left(\frac{\pi}{D-3}\right)^{2} M_{D-2}^{D-2}\left(\ell M_{D-2}\right)^{2}\left(\frac{M_{D}}{M_{D-2}}\right)^{2 D-4} .
$$


In our primary case of interest, of a minimal (simple) supersymmetry-preserving compactification to $D=6$ dimensions, this becomes

$$
\Lambda \sim \frac{\Delta \omega^{2}}{\ell^{2}}=\frac{\pi^{2}}{9} \ell^{2} \frac{M_{6}^{8}}{M_{4}^{2}}, \quad \stackrel{1 / \ell \sim M_{4} \mapsto M_{P}}{M_{6} \mapsto M} \quad M_{\Lambda} \sim M^{2} / M_{P}
$$

and relates the mass scales of the vacuum energy/cosmological constant $\left(M_{\Lambda}\right)$, particle physics/Standard Model $\left(M_{6} \mapsto M\right)$, and the Planck scale $\left(M_{P}\right)$.

More precisely, the $D$ - and the $(D-2)$-dimensional characteristic (Planck) mass scales are related by the exact expression $[30,31,16]^{3}$

$$
M_{D-2}^{D-4}=M_{D}^{D-2} 2 \pi \ell^{2}\left|z_{0}\right|^{-\frac{D-1}{2(D-2)}} e^{z_{0}} \Gamma_{ \pm}\left(\frac{D-3}{2(D-2)} ; \frac{1}{\left|z_{0}\right|}\right),
$$

where $z_{0}$ is the radial distance (in units of $\ell$ ) from the $(D-2)$ dimensional brane world to the boundary of $\mathscr{Y}_{\perp}^{2}$, and $\Gamma_{ \pm}$denotes the "[0, $\left.\frac{1}{\left|z_{0}\right|}\right]$-incomplete gamma function" for $z_{0}<0$ and its complement for $z>0$. For $D=6$, this yields

$$
M_{4}^{2}=\zeta_{0}\left|z_{0}\right|^{-\frac{5}{8}} e^{z_{0}} M_{6}^{4} \ell^{2}, M_{4}=\sqrt{\zeta_{0}}\left|z_{0}\right|^{-\frac{5}{16}} e^{z_{0} / 2} \frac{M_{6}^{2}}{M_{\ell}}
$$

where $0 \leqslant \zeta_{0} \stackrel{\text { def }}{=} 2 \pi \Gamma\left(\frac{3}{8} ; \frac{1}{\left|z_{0}\right|}\right) \leqslant 2 \pi \Gamma\left(\frac{3}{8}\right) \approx 14.89$,

focusing on the $z_{0}>0$ case of (30), since that enables the $e x$ ponential hierarchy $M_{4} \gg M_{6} \cdot{ }^{4}$ In turn, solving (31) for $M_{6}$, the cosmological constant (29) becomes

$$
\Lambda \sim \frac{\pi^{2}}{9 \zeta_{0}}\left|z_{0}\right|^{5 / 4} e^{-2 z_{0}} \frac{M_{4}^{2}}{\ell^{2}}=\frac{\pi^{2}}{9 \zeta_{0}}\left|z_{0}\right|^{5 / 4} e^{-2 z_{0}} M_{4}^{2} M_{\ell}^{2},
$$

which reveals the effect of the $z_{0}$-driven exponential hierarchy in the "axilaton" models $[30,31,32,33,16]$. Therefore, the primordial "size-of-the-universe-scale" $L$ in (19)-(21) is free to be naturally within one or two orders of magnitude of the stringy fundamental length scale $\lambda$, resulting in a phenomenologically realistic scale introduced by the mixing terms (19)-(21).

The seesaw expression appears to be technically natural. That is, when $M_{P} \rightarrow \infty$, the cosmological constant scale goes to zero, and in that case, the dual space curvature is zero, and we get a flat dual space and thus enhanced symmetry. This is precisely what 't Hooft naturalness requires: when the physical cutoff in some theory goes to infinity, the small parameters in the theory vanish and should be protected by some hidden symmetry. It is tempting to relate that hidden symmetry to supersymmetry. However, this appears to be a bit too naive. Such conjectural supersymmetry restoration requires the vanishing of the Ricci tensor, which requires $\omega \rightarrow 0$ : the vanishing

\footnotetext{
${ }^{3}$ Note: the exponential factor [31, Eq. (15)] was inadvertently omitted in [16, Eq. (3.3)]. Also, note that $0 \leqslant \Gamma(x ; y) \stackrel{\text { def }}{=}(\Gamma(x)-\gamma(x ; y)) \leqslant \Gamma(x)$, and both incomplete gamma functions range from 0 to $\Gamma(x)$.

${ }^{4}$ In the "naked singularity to brane-world" coalescing limit $\lim _{z_{0} \rightarrow 0} \Gamma\left(\frac{3}{8} ; \frac{1}{\left|z_{0}\right|}\right)\left|z_{0}\right|^{-5 / 8} e^{z_{0}}=0$ since $\Gamma\left(\frac{3}{8} ; \frac{1}{\left|z_{0}\right|}\right)$ vanishes faster than any (negative) power can diverge. In turn, moving the naked singularity away from the brane-world by keeping $z_{0} \neq 0$ makes the hierarchy grow exponentially, $\sim e^{z} 0$.
}

$\Lambda \propto \Delta \omega^{2} \rightarrow 0$ is necessary, but not sufficient. Letting $\Lambda \rightarrow 0$ by sending $M_{4} \rightarrow \infty$ can be forced by letting $z_{0} \rightarrow \infty$ in (31), the geometrical meaning of which is that $\mathscr{Y}_{\perp}^{2} \rightarrow S^{1}$ "at infinity," indicating some singular dimensional collapse.

This seesaw formula can be rewritten in a form that is even more appealing by relating the scale $M$ to the Planck scale via an exponential factor $M=M_{p} \exp \left(-\right.$ const. $\left.M_{p} / M_{i}\right)$, and thus the vacuum energy density $M_{p}^{2} \Lambda$ is given as

$$
M_{\Lambda}^{4}=M_{p}^{4} \exp \left\{-8 \text { const. } M_{p} / M_{i}\right\},
$$

where $M_{i}$ correspond to what is roughly an effective scale that numerically corresponds to the inflation scale (see also [3]). Note that (33) comes close to this, except that it is hard to set $z_{0} \rightarrow M_{4} / M_{i}$ except by hand. Nevertheless, our toy model, even though realized in the conventional spacetime interpretation of string theory, does illustrate the main features of the generic noncommutative and doubled formulation of string theory, at least when it comes to the dark energy seesaw and its relation to the separation of scales associated with the hierarchy problem. Finally, we comment on the UV and IR scales in the section on dark matter and string theory. The UV (or the noncommutativity scale) should be considered as an effective scale to be empirically determined. In turn, $L$ is the primordial IR cutoff.

\section{A NONPERTURBATIVE FORMULATION OF STRING THEORY}

In view of the preceding discussion regarding the generic formulation of string theory and its relation to the problem of dark energy and dark matter, we now propose a nonperturbative formulation of string theory (and its M- and F-theory avatars). Indeed, the chiral string worldsheet theory offers such a new view on the fundamental question of a nonperturbative formulation of quantum gravity [14] by noting the following: in the chiral string worldsheet description, the target space is found to be a modular space (quantum spacetime), but the same can be also said of the worldsheet. If the string worldsheet is made modular in its chiral formulation, by doubling of $\tau$ and $\sigma$, so that $\mathbb{X}(\tau, \sigma) \rightarrow \widehat{\mathbb{X}}(\tau, \sigma)$ can be in general viewed as an infinitedimensional matrix (acting on the basis of Fourier components of $\tilde{\tau}$ and $\tilde{\sigma}$, the doubles of $\tau$ and $\sigma$, respectively), then the corresponding chiral string worldsheet action becomes

$$
\int_{\tau, \sigma} \operatorname{Tr}\left[\partial_{\tau} \widehat{\mathbb{X}}^{A} \partial_{\sigma} \widehat{\mathbb{X}}^{B}\left(\omega_{A B}+\eta_{A B}\right)-\partial_{\sigma} \widehat{\mathbb{X}}^{A} H_{A B} \partial_{\sigma} \widehat{\mathbb{X}}^{B}\right],
$$

where the trace is over the (suppressed) matrix indices. The matrix elements then emerge as the natural partonic degrees of freedom. We arrive at a nonperturbative quantum gravity by replacing the $\sigma$-derivative with a commutator involving one extra $\widehat{\mathbb{X}}^{26}$ (with $\left.A=0,1,2, \ldots, 25\right)^{5}$ :

$$
\partial_{\sigma} \widehat{\mathbb{X}}^{A} \rightarrow\left[\widehat{\mathbb{X}}^{26}, \widehat{\mathbb{X}}^{A}\right]
$$

${ }^{5}$ That the canonical worldsheet of string theory might become noncommutative in a deeper, nonperturbative formulation, was suggested in [6]. 
This dictionary suggests the following fully interactive and nonperturbative formulation of the chiral string worldsheet theory in terms of an (M-theory-like) matrix model form of the above chiral string action (with $a, b, c=0,1,2, \ldots, 25,26$ )

$$
\int_{\tau} \operatorname{Tr}\left[\partial_{\tau} \widehat{\mathbb{X}}^{a}\left[\widehat{\mathbb{X}}^{b}, \widehat{\mathbb{X}}^{c}\right] \eta_{a b c}-H_{a c}\left[\widehat{\mathbb{X}}^{a}, \widehat{\mathbb{X}}^{b}\right]\left[\widehat{\mathbb{X}}^{c}, \widehat{\mathbb{X}}^{d}\right] H_{b d}\right],
$$

where the first term is of a Chern-Simons form and the second of the Yang-Mills form, and $\eta_{a b c}$ contains both $\omega_{A B}$ and $\eta_{A B}$. This is then the nonperturbative "gravitization of the quantum" [14]. We remark that in this nonperturbative matrix theory-like formulation of the chiral string (and quantum gravity), the matrices emerge from the modular worldsheet, and the fundamental commutator from the Poisson bracket with respect to the dual worldsheet coordinates (of the modular/quantum worldsheet) — that is, quantum gravity "quantizes" itself, and thus quantum mechanics originates in quantum gravity. (However, this formulation should be distinguished from Penrose's "gravitization of the quantum" and gravity-induced "collapse of the wave function" [38]. Also, note some similarity of the chiral string worldsheet formulation, in its intrinsic noncommutative form, to the most recent proposal by Penrose regarding "palatial" twistor theory [39].)

At this point, we also recall that the authors of [6] explicitly state in the conclusion of their paper the following. "(1) The density of gauge invariant degrees of freedom, per unit energy, per unit spacetime volume, is much less in the proper formulation of string field theory than in any ordinary relativistic field theory... (2) The translation degree of freedom of the string center of mass is in some sense doubled... (3) The familiar continuous world sheets should be replaced, in the proper formulation of the classical theory, by some less continuous structure, perhaps related to continuum worldsheets the way quantum phase space is related to classical phase space." This prescient prediction is remarkably close to our nonperturbative formulation.

In thinking about nonperturbative matrix model formulations of string theory, it is natural to invoke the IIB matrix model [40], based on D-instantons as well as the matrix model of M-theory [41], based on D0-branes. However, these matrix models lack very important covariant properties associated with F-theory and M-theory. In our proposal, we can do better. Given our new viewpoint, we can suggest a new covariant noncommutative matrix model formulation of F-theory, by also writing in the large $N$ limit $\partial_{\tau} \widehat{\mathbb{X}}^{C}=\left[\widehat{\mathbb{X}}, \widehat{\mathbb{X}}^{C}\right]$, in terms of commutators of two (one for $\partial_{\sigma} \widehat{\mathbb{X}}^{C}$ and one for $\partial_{\tau} \widehat{\mathbb{X}}^{C}$ ) extra $N \times N$ matrix-valued chiral $\widehat{\mathbb{X}}^{\prime}$ s. Notice that, in general, we do not need an overall trace, and so the action can be viewed as a matrix, rendering the entire nonperturbative formulation of F-theory as purely quantum in the sense of the original matrix formulation of quantum mechanics by Born-Jordan and BornHeisenberg-Jordan [42]

$$
\mathrm{S}_{\mathrm{ncF}}=\frac{1}{4 \pi}\left[\widehat{\mathbb{X}}^{a}, \widehat{\mathbb{X}}^{b}\right]\left[\widehat{\mathbb{X}}^{c}, \widehat{\mathbb{X}}^{d}\right] f_{a b c d},
$$

where instead of 26 bosonic $\widehat{\mathbb{X}}$ matrices one would have 28, with supersymmetry emerging in $10(+2)$ dimensions from this underlying bosonic formulation. This formulation realizes the $S L(2, \mathbf{Z})$ symmetry of IIB string theory. In this noncommutative matrix model formulation of F-theory, in general, $f_{a b c s}(\widehat{\mathbb{X}})$ is a dynamic background, determined by the matrix analog of the vanishing of the relevant beta function. By T-duality, the new covariant M-theory matrix model reads as

$$
\mathrm{S}_{\mathrm{ncM}}=\frac{1}{4 \pi} \int_{\tau}\left(\partial_{\tau} \widehat{\mathbb{X}}^{i}\left[\widehat{\mathbb{X}}^{j}, \widehat{\mathbb{X}}^{k}\right] g_{i j k}-\left[\widehat{\mathbb{X}}^{i}, \widehat{\mathbb{X}}^{j}\right]\left[\widehat{\mathbb{X}}^{k}, \widehat{\mathbb{X}}^{l}\right] h_{i j k l}\right),
$$

with 27 bosonic $\widehat{\mathbb{X}}$ matrices, with supersymmetry emerging in 11 dimensions. Once again, the backgrounds $g_{i j k}(\widehat{\mathbb{X}})$, and $h_{i j k l}(\widehat{\mathbb{X}})$ are fully dynamical and should be determined by a matrix-analog of the Renormalization Group (RG) equation and the vanishing of the corresponding beta function.

Note that in this approach holography [43] (such as AdS/CFT [44], which can be viewed as a "quantum Jarzynski equality on the space of geometrized RG flows" [45]) is emergent in a particular semiclassical "extensification" of quantum spacetime, in which the dual spacetime degrees of freedom are also completely decoupled. The relevant information about $\omega_{A B}, \eta_{A B}$, and $H_{A B}$ is now contained in the new dynamical backgrounds $f_{a b c d}$ in F-theory and $g_{i j k}$ and $h_{i j k l}$ in M-theory. This proposal offers a new formulation of covariant Matrix theory in the M-theory limit [46], which is essentially a partonic formulation: strings emerge from partonic constituents in a certain limit. This new matrix formulation is fundamentally bosonic, and thus, it is reminiscent of bosonic M-theory [47]. The relevant backgrounds $g_{i j k}$ and $h_{i j k l}$ should be determined by the matrix RG equations. Also, there are lessons here for the new concept of "gravitization of quantum theory" as well as the idea that dynamical Hilbert spaces or 2-Hilbert spaces (here represented by matrices) are fundamentally needed in quantum gravity [48]. This matrix-like formulation should be understood as a general nonperturbative formulation of string theory. In this partonic (quantum spacetime) formulation, closed strings (as well as branes) are collective excitations, in turn constructed from the product of open string fields. Similarly, our toy model can be understood as a collective excitation in this more fundamental "partonic" formulation. The observed classical spacetime emerges as an "extensification" [14], in a particular limit, out of the basic building blocks of quantum spacetime. Their remnants can be found in the low-energy bilocal quantum fields, with bilocal quanta, which were a motivation for our discussion of dark matter in string theory.

Finally, it is an old realization that the 10d superstring can be found as a solution of the bosonic string theory [5]. The authors [5] explicitly state in their abstract that "consistent closed ten-dimensional superstrings, i.e., the two $N=1$ heterotic strings and the two $N=2$ superstrings, are contained in the 26-dimensional bosonic closed string theory. The latter thus appears as the fundamental string theory." This is precisely what we have in our proposed nonperturbative formulation. (Such a bosonic formulation is also endowed with higher mathematical symmetries, as already observed in [8].) Supersymmetry (in the guise of M- and F-theory) is emergent from 
our nonperturbative and seemingly entirely bosonic formulation in a similar fashion. This should allow going around the obvious problems raised by the apparent falsification of supersymmetry at the observable LHC energies.

\section{CONCLUDING REMARKS}

In this paper, we have related the problems of dark energy and dark matter to the hierarchy problem, in the context of a general noncommutative formulation of string theory, wherein dark energy is generated by the dynamical geometry of dual spacetime. In particular, dark matter stems from the degrees of freedom dual to the visible matter. This generic formulation of string theory is sensitive to both the IR and UV scales. The Standard Model (Higgs) scale is radiatively stable by being a geometric mean of these two radiatively stable scales, which clearly goes beyond the reach of effective field theory. We also have commented on various phenomenological signatures of this new approach to dark energy, dark matter, and the hierarchy problem in the context of string theory and the realization of this new view on the hierarchy problem within a discretuum of toy models based on a nonholomorphic deformation of stringy cosmic strings. Finally, we have presented a proposal for a new nonperturbative formulation of string theory, which sheds light on both M- and F-theory, and illuminates issues related to supersymmetry and holography.

In conclusion, we point out that the sequester mechanism discussed in this paper can be used to stabilize the moduli and that it offers a new view on SUSY breaking beyond effective field theory, based on T-duality and intrinsic noncommutativity of string theory. In our approach, SUSY might be important for fixing the zero value of the cosmological constant only in the limit of infinite Planck scale in $4 \mathrm{~d}$ and, also, for the stability of locally emergent Minkowski spacetime. Also, in our approach, the Standard Model does not have to be realized via the KaluzaKlein mechanism and string compactifications, but in the context of "extensification" of the nonpertubative formulation of chiral string worldsheet theory, in which case, one should look for robust noncommutative structures in the Standard Model, as indicated by Connes approach to the so-called noncommutative geometry of the Standard Model [49] and its phenomenology [50,51,52]. This new view of string theory should bring about a different viewpoint on the vacuum selection problem (along the lines of the attractor solutions found in [53]), as well as the selection of a robust quantum matter sector which is mutually consistent with the quantum gravitational sector and their respective duals.

\section{CONFLICTS OF INTEREST}

The authors declare that there are no conflicts of interest regarding the publication of this paper.

\section{ACKNOWLEDGMENTS}

We would like to thank J. A. Argyriadis, D. Edmonds, L. Freidel, V. Jejjala, M. Kavic, J. Kowalski-Glikman, Y.-H. He, R. Leigh, and T. Takeuchi for discussions. PB would like to thank the Simons Center for Geometry and Physics and the CERN Theory Group, for their hospitality, and TH is grateful to the Department of Physics, University of Maryland, and the Physics Department of the University of Novi Sad, Serbia, for hospitality and resources. The work of DM is supported in part by the Department of Energy (under DOE grant number DESC0020262) and the Julian Schwinger Foundation. DM is grateful to Perimeter Institute for hospitality and support.

\section{References}

[1] J. Polchinski, String theory. Vol. 1 and Vol. 2 . Cambridge University Press, 2007.

[2] M. Grana, Phys. Rept. 423 (2006) 91; M. R. Douglas and S. Kachru, Rev. Mod. Phys. 79 (2007) 733; S. R. Green et al. Class. Quant. Grav. 29 (2012) 075006; D. Kutasov et al. Phys. Rev. Lett. 115, no. 7 (2015) 071305; U. H. Danielsson and T. Van Riet, Int. J. Mod. Phys. D27 no. 12, (2018) 1830007.

[3] G. Obied, H. Ooguri, L. Spodyneiko, and C. Vafa, arXiv:1806.08362 [hep-th]. P. Agrawal, G. Obied, P. J. Steinhardt, and C. Vafa, Phys. Lett. B784 (2018) 271-276.

[4] C. Vafa, arXiv:hep-th/0509212; D. Andriot, Fortsch. Phys. 67, no.7, 1900026 (2019). E. Palti, Fortsch. Phys. 67, no.6, 1900037 (2019).

[5] A. Casher, F. Englert, H. Nicolai and A. Taormina, Phys. Lett. B 162, 121-126 (1985)

[6] J. J. Atick and E. Witten, Nucl. Phys. B 310, 291 (1988).

[7] A. A. Tseytlin, Phys. Lett. B 242 (1990) 163-174. Nucl. Phys. B 350 (1991) 395-440. Phys. Rev. Lett. 66 (1991) 545-548.

[8] G. W. Moore, "Finite in all directions," arXiv:hep-th/9305139.

[9] M. R. Douglas and N. A. Nekrasov, Rev. Mod. Phys. 73, 977 (2001); R. J. Szabo, Phys. Rept. 378, 207 (2003); H. Grosse and R. Wulkenhaar, Commun. Math. Phys. 256, 305 (2005).

[10] W. Siegel, Phys. Rev. D 475453 (1993); Phys. Rev. D 48, 2826 (1993) C. Hull, and B. Zwiebach, JHEP 0909099 (2009); O. Hohm, C. Hull and B. Zwiebach, JHEP 1007, 016 (2010); O. Hohm, D. Lust and B. Zwiebach, Fortsch. Phys. 61, 926 (2013).

[11] G. Amelino-Camelia, L. Freidel, J. Kowalski-Glikman and L. Smolin, Phys. Rev. D 84, 084010 (2011); Gen. Rel. Grav. 43, 2547 (2011) [Int. J. Mod. Phys. D 20, 2867 (2011)].

[12] Y. Aharonov, and D. Rohrlich, Quantum Paradoxes: Quantum Theory for the Perplexed (New York: Wiley) (2005).

[13] D. Minic and H. C. Tze, Phys. Lett. B 581, 111-118 (2004); Phys. Rev. D 68, 061501 (2003); V. Jejjala, M. Kavic and D. Minic, Int. J. Mod. Phys. A 22, 3317-3405 (2007). 
[14] L. Freidel, R. G. Leigh, and D. Minic, Phys. Lett. B 730 (2014) 302-306. Int. J. Mod. Phys. D 23 (12), (2014) 1442006. JHEP 06 (2015) 006. Int. J. Mod. Phys. D 24 (12), (2015) 1544028. Phys. Rev. D 94 (10), (2016) 104052. J. Phys. Conf. Ser. 804 (1) (2017) 012032. Int. J. Mod. Phys. A 34 (28), (2019) 1941004. JHEP 09 (2017) 060. Phys. Rev. D 96 (6) (2017) 066003. L. Freidel, J. Kowalski-Glikman, R. G. Leigh, and D. Minic, Phys. Rev. D 99 (6) (2019) 066011. D. Minic, [arXiv : 2003.00318 [hep-th]].

[15] P. Berglund, T. Hubsch and D. Minic, Phys. Lett. B 798 (2019) 134950. Int. J. Mod. Phys. D 28 (14), (2019) 1944018.

[16] P. Berglund, T. Hubsch and D. Minic, JHEP 19 (2020) 166.

[17] D. H. Friedan, Ann. Phys. 163 (1985) 318-419. Phys. Rev. Lett. 45 (1980) 1057.

[18] C. M. Ho, D. Minic, and Y. J. Ng, Phys. Lett. B 693 (2010) 567-570. Gen. Rel. Grav. 43 (2011) 2567-2573. Phys. Rev. D 85 (2012) 104033. D. Edmonds, D. Farrah, C. M. Ho, D. Minic, Y. J. Ng, and T. Takeuchi, Astrophys. J. 793 (2014) 41. Int. J. Mod. Phys. A 32 (18), (2017) 1750108.

D. Edmonds, D. Farrah, D. Minic, Y. J. Ng, and T. Takeuchi, Int. J. Mod. Phys. D 27 (02), (2017) 1830001.Bulg. J. Phys. 45 (2), (2018) 138-151; D. Edmonds, D. Minic and T. Takeuchi, arXiv: 2005.08927 [astro-ph.C0], to appear in IJMPD; arXiv:2009.12915 [astro-ph.c0].

[19] A. Davidson and S. Rubin, Class. Quant. Grav. 26, (2009) 235019. Phys. Rev. D 89 (2014) 024036.

[20] N. Craig and S. Koren, JHEP 03, 037 (2020).

[21] N. Kaloper and A. Padilla, Phys. Rev. Lett. 112 (9), (2014) 091304.

[22] N. Kaloper and A. Padilla, Phys. Rev. D 90 (8), (2014) 084023; [ibid. (10), (2014) 109901]. A. Padilla, “Lectures on the Cosmological Constant Problem," arXiv:1502.05296 [hep-th].

[23] L. Freidel, J. Kowalski-Glikman, R. G. Leigh, and D. Minic, in preparation.

[24] E. Witten, Nucl. Phys. B403 (1993) 159-222, hep-th/9301042.

[25] C. F. Doran, M. G. Faux, S. J. Gates, Jr., T. Hübsch, K. M. Iga, and G. D. Landweber, J. Phys. A 42 (2008) 065402, arXiv:0803.3434 [hep-th].

[26] J. Polonyi, "Generalization of the massive scalar multiplet coupling to the supergravity,". Hungarian Central Institute for Research in Physics pub.: KFKI-77-93.

[27] S. V. Ketov and M. Y. Khlopov, Bled Workshops Phys. 19 (2), (2018) 148-163. Eur. Phys. J. C 79 (8), (2019) 713.

[28] L. Verde, T. Treu and A. G. Riess, "Tensions between the Early and the Late Universe," arXiv:1907.10625 [astro-ph.CO].

[29] V. Jejjala, M. Kavic, D. Minic and T. Takeuchi, in preparation.

[30] P. Berglund, T. Hubsch, and D. Minic, JHEP 09 (2000) 015. JHEP 02 (2001) 010. JHEP 01 (2001) 041.

[31] P. Berglund, T. Hübsch, and D. Minic, Phys. Lett. B 512 (2001) 155-160.
[32] P. Berglund, T. Hübsch, and D. Minic, Phys. Lett. B 534 (2002) 147-154.

[33] P. Berglund, T. Hübsch, and D. Minic, Phys. Rev. D 67 (2003) 041901.

[34] C. Vafa, Nucl. Phys. B 469, 403-418 (1996).

[35] A. G. Cohen and D. B. Kaplan, Phys. Lett. B 470, 52-58 (1999).

[36] B. R. Greene, A. D. Shapere, C. Vafa and S. T. Yau, Nucl. Phys. B 337, 1-36 (1990).

[37] J. Polchinski and A. Strominger, Phys. Rev. Lett. 67 (1991) 1681.

[38] R. Penrose, Found. Phys. 44, 557 (2014).

[39] R. Penrose, Phil. Trans. Roy. Soc. Lond. A 373, 20140237 (2015).

[40] N. Ishibashi, H. Kawai, Y. Kitazawa, and A. Tsuchiya, Nucl. Phys. B498 (1997) 467-491.

[41] T. Banks, W. Fischler, S. H. Shenker, and L. Susskind, Phys. Rev. D55 (1997) 5112-5128.

[42] B. L. van der Waerden, Sources of Quantum Mechanics (New York: Dover) (1968).

[43] G. 't Hooft, Conf. Proc. C 930308, 284 (1993); L. Susskind, J. Math. Phys. 36, 6377 (1995).

[44] J. M. Maldacena, Int. J. Theor. Phys. 38, 1113 (1999) [Adv. Theor. Math. Phys. 2, 231 (1998)]; S. S. Gubser, I. R. Klebanov and A. M. Polyakov, Phys. Lett. B 428, 105 (1998); E. Witten, Adv. Theor. Math. Phys. 2, 253 (1998).

[45] D. Minic and M. Pleimling, Phys. Lett. B 700, 277 (2011); N. Gray, D. Minic and M. Pleimling, Int. J. Mod. Phys. A 28, 1330009 (2013).

[46] D. Minic, arXiv:hep-th/9909022; arXiv:hep-th/0009131. H. Awata, M. Li, D. Minic and T. Yoneya, JHEP 0102, 013 (2001). D. Minic and H. C. Tze, Phys. Lett. B 536, 305 (2002);

[47] G. T. Horowitz and L. Susskind, J. Math. Phys. 42, 3152 (2001).

[48] S. Bunk, L. Muller and R. J. Szabo, Lett. Math. Phys. 109, no. 8, 1827 (2019); V. Balasubramanian, J. de Boer and D. Minic, arXiv:gr-qc/0211003; L. Smolin, J. Math. Phys. 36, 6417 (1995); L. Crane, J. Math. Phys. 36, 6180 (1995). D. Minic, Phys. Lett. B 442, 102 (1998); T. Banks and W. Fischler, arXiv: hep-th/0102077.

[49] A. Connes, Noncommutative Geometry, Academic Press, San Diego, 1994; A. Connes and M. Marcolli, Noncommutative Geometry, Quantum Fields and Motives, AMS, 2007.

[50] A. H. Chamseddine and W. D. Van Suijlekom, "A survey of spectral models of gravity coupled to matter," arXiv:1904.12392 [hep-th].

[51] A. Devastato, M. Kurkov and F. Lizzi, Int. J. Mod. Phys. A 34 (19), (2019) 1930010.

[52] U. Aydemir, D. Minic and T. Takeuchi, Phys. Lett. B 724 (2013) 301. U. Aydemir, D. Minic, C. Sun and T. Takeuchi, Phys. Rev. D 91 (2015) 045020. Int. J. Mod. Phys. A 31 (01), (2016) 1550223. Mod. Phys. Lett. A 31 (18), (2016) 1650101. JHEP 1809 (2018) 117. 
[53] J. A. Argyriadis, Y. H. He, V. Jejjala and D. Minic, "Dynamics of genetic code evolution: The emergence of universality," arXiv:1909.10405 [q-bio.0T], to appear in PRE. 